\title{
L'embâcle de bois en rivière : un bienfait écologique ? un facteur de risques naturels ?
}

\author{
par L. Maridet a, H. Piégay ${ }^{\mathrm{b}}$, O. Gilard ${ }^{\mathrm{c}}$ et A. Thévenet ${ }^{\mathrm{a}}$
}

\section{INTRODUCTION}

Dans cet article, nous définirons l'embâcle comme étant une accumulation hétérogène de bois mort façonnée par les écoulements ; tout autre objet (matière plastique, pneu, épaves, tissu) susceptible de s'entasser également dans un cours d'eau n'entrant pas, bien sûr, dans la définition précédente. Ces amas de débris ligneux proviennent de la végétation rivulaire adjacente ou ripisylve. Réservoir potentiel d'embâcle, ces formations végétales riveraines influeront d'autant plus sur le fonctionnement du cours d'eau que ceux-ci sont de faible largeur.

Dans le cadre de la politique générale de protection contre les risques d'inondation et d'érosion, et face aux épisodes pluviométriques exceptionnels de ces dernières années à l'origine de catastrophes, la formation des embâcles a été vivement combattue par les services de l'Etat. Afin de préserver la capacité d'écoulement dans le chenal, ces amas de débris ligneux ont été et sont encore systématiquement éliminés. Pourtant, même si elles ont des incidences hydrauliques et morphologiques, ces structures ont un rôle prépondérant sur le fonctionnement global des cours d'eau. Localisées en berge ou dans le chenal, les accumulations de bois mort agiront sur le fonctionnement hydraulique, morphologique et biologique du cours d'eau. La localisation et

a CEMAGREF, Division Biologie des Ecosystèmes Aquatiques, Laboratoire Hydroécologie Quantitative, 3 bis, quai Chauveau, 69336 Lyon Cedex 09. France.

"UMR 5600 "Environnement, ville, société". 18, rue Chevreul, 69362 Lyon Cedex 07, France.

CEMAGREF, Division Hydrologie Hydratulque, 3 bis, quai Chauveau, 69336 Lyon Cedex 09, France. la position de ces débris ligneux, le style géomorphologique, la taille ou le degré de domestication du cours d'eau, la nature du corridor végétal seront autant de paramètres qui influeront sur le fonctionnement.

Dans cette synthèse, nous aborderons successivement la notion de risque lié à la formation d'embâcles puis leur rôle écologique. Enfin, quelques principes de gestion de ces structures ligneuses seront proposés.

\section{L'EMBÂCLE: UN FACTEUR DE RISQUE?}

\subsection{Le fonctionnement hydraulique d'un embâcle}

Les embâcles se forment généralement dans les lits mineurs des cours d'eau lorsqu'un obstacle vient favoriser l'accumulation des débris ligneux. Ils constituent localement un barrage, ou, tout au moins, un frein à l'écoulement, qui se traduit par une perte de charge localisée dont l'importance dépend du volume de matériau qui le constitue (fig. 1). Physiquement, cette perte de charge se concrétise (fig. 2):

- en amont de l'obstacle par une élévation du niveau d'eau, une réduction des vitesses de courant et une sédimentation des particules minérales liée à la diminution des forces érosives ;

- en aval, en raison du laminage, par la réduction du débit de pointe.

Lorsque l'embâcle n'occupe pas tout le lit mineur du cours d'eau, la perte de charge localisée se traduit au contraire par une concentration de l'écoulement avec une augmentation des vitesses et des forces tractrices dans une partie seulement de la section en travers (fig. 2a). Ainsi,

In order to preserve the stream flow capacity, the French Government always recommends the cleaning of riparian vegetation and the removing of coarse woady debris in the channel. But, these management practices contrast with the ecological role of the coarse woody debris and the small risk of these structures in rural area. The «do nothing ", "rehabilitation » and "localized manipulation " notions appear now in different management options. In this paper; we propose a synthesis on the risk due to coarse woody debris (inundation and erosion) and on their ecological role. We conclude the article by proposing a few management recommendations of these woody structures. 


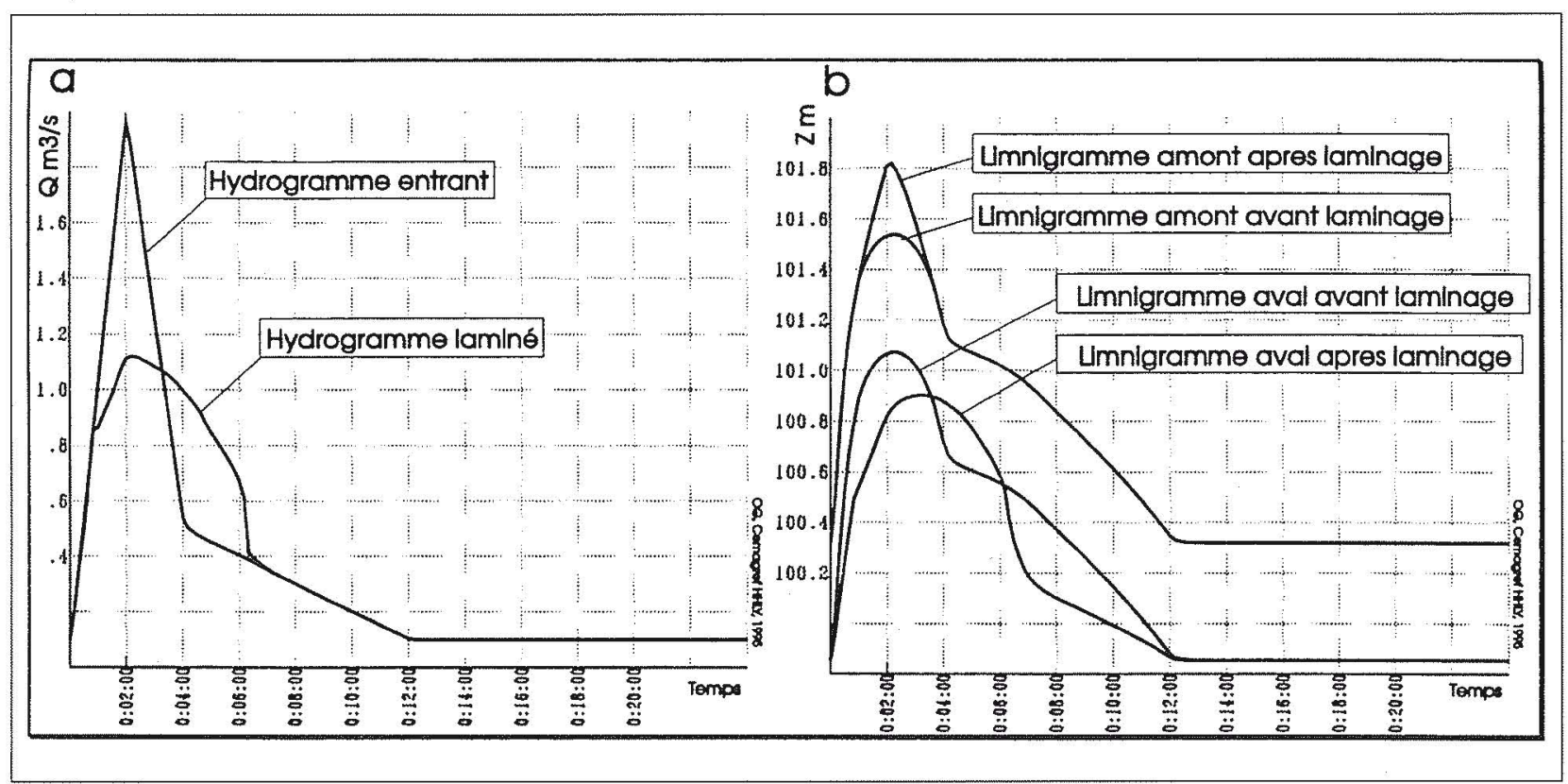

1. Simulation par modèle numérique d'un phénomène de laminage :

- a : Débit en fonction du temps $(Q(t))$,

- b : Cote en fonction du temps $(Z(t))$.

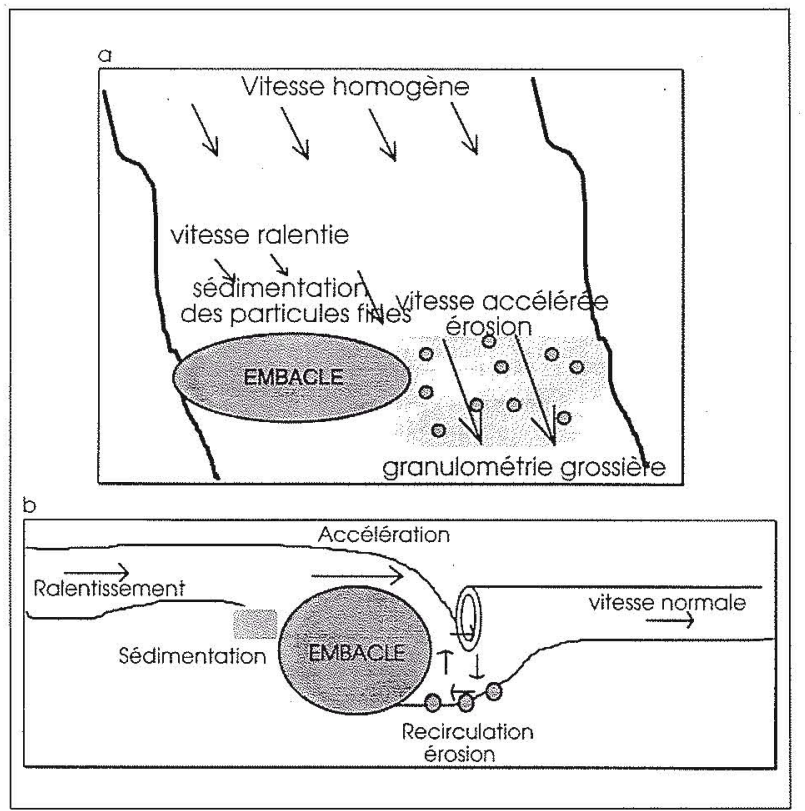

2. La répartition schématique des vitesses :

- a : dans une section en travers (vue en plan),

- b : en cas de surverse (coupe).

juste en amont de l'obstacle, la réduction des vitesses entraîne une sédimentation de particules fines (dépôt des sables et des limons) alors que, dans la partie "ouverte" de la section, l'accroissement des vitesses favorise l'enlèvement des fines pour laisser en place une granulométrie plus grossière.

Lorsque les matériaux sont à moitié submergés ou lors des épisodes de crue, les embâcles peuvent jouer le rôle de déversoir. Il se produit alors juste à l'aval de ce déversoir une fosse de dissipation de l'énergie, c'est-à-dire un affouillement qui peut atteindre quelques décimètres de profondeur ( fig. $2 b$ ). On observe également dans cette situation d'eau une grande hétérogénéité des vitesses avec une recirculation et des tourbillons au niveau de la fosse, une mise en vitesse au-dessus de l'obstacle et une réduction en amont. Là encore, les processus de transport solide associés aux forces tractrices entrainnent une hétérogénéité du substrat, les particules fines étant plus fréquentes en amont et les particules grossières plus apparentes à l'aval immédiat de l'obstacle.

Si les appuis sur lesquels s'est formé l'embâcle sont peu résistants (stabilité de l'embâcle) il peut se produire en fortes eaux une rupture d'embâcle qui se traduit hydrauliquement par le fonctionnement inverse du précédent: il y a alors un déstockage brutal du volume d'eau concerné, avec un brusque abaissement du niveau d'eau à l'amont du point considéré et inversement une augmentation du débit à l'aval. L'ampleur du phénomène dépend bien entendu de sa brutalité, qui elle-même dépend de la dynamique de la rupture, et du volume de liquide concerné.

\subsection{Embâcles et risques naturels}

\subsubsection{Le risque d'inondation}

Le fonctionnement hydraulique décrit précédemment a une incidence sur le risque d'inondation, mais il convient d'être prudent dans son interprétation. En effet, lorsque l'embâcle joue son rôle de frein, on constate une augmentation du niveau d'eau à l'amont, qui se traduit par un risque accru uniquement si l'occupation du sol de la zone concernée est sensible à l'inondation. Ainsi, dans des situations très particulières, l'apparition d'un embâcle peut avoir des conséquences désastreuses : c'est le cas des obstructions d'ouvrages hydrauliques en zone urbaine fortement vulnérable aux inondations. En effet, suite à des accumulations de débris flottants contre des piles de ponts, le risque d'inondation peut être fortement aggravé en favorisant les débordements hors du lit. Heureusement, le plus souvent, les embâcles se forment dans des endroits qui ne pâtissent guère d'une aggravation de quelques décimètres du niveau d'eau et où la végétation est abondante (friches, forêts). De plus, lors 
d'une expérimentation en laboratoire, Young ne note une élévation significative de la ligne d'eau qu'en présence de fortes accumulations de bois.

Inversement, les processus de laminage induits par le stockage d'un certain volume d'eau, en réduisant les débits, diminuent le risque à I'aval en modifiant deux caractéristiques de l'hydrogramme sortant: d'abord le débit maximum atteint, mais aussi la vitesse de montée des eaux. En effet, on ne peut pas dissocier les phénomènes de stockage de ceux de ralentissement, dont l'incidence devient parfois primordiale en accordant un "délai de grâce" supplémentaire aux zones situées à l'aval.

Le risque principal se rencontre lors des ruptures d'embâcles, dont la brutalité peut avoir des conséquences dommageables. Mais, en France, les volumes de bois concernés sont en général faibles. L'incidence d'une rupture est donc limitée aux quelques dizaines de mètres situés à l'aval, l'amortissement d'une onde de crue très brutale étant aussi très rapide. De plus, la probabilité d'avoir une rupture simultanée de plusieurs embâcles stockant de petites quantités d'eau est très faible. Ainsi, la réduction des débits lors de la constitution de ces embâcles et l'augmentation des temps de transfert de la crue dans le réseau hydrographique peuvent l'emporter assez largement sur les effets négatifs observés lors d'une rupture. Il est cependant très hasardeux de quantifier ces phénomènes, sauf pour des événements moyens, car une multitude de paramètres mal connus entre en considération: la répartition spatio-temporelle de l'événement pluvieux, l'état de la surface des sols, la vitesse de constitution de l'embâcle, le volume de celui-ci. Par ailleurs ces paramètres varient d'un événement à l'autre.

\subsubsection{Risque d'érosion ou stabilisation?}

Comme le risque d'inondation, l'influence des embâcles sur les phénomènes d'érosion doit être analysée dans toute sa complexité. Associer intrinsèquement risque d'érosion et embâcle constitue une gageure. En effet, ce risque diffère d'une rivière à l'autre en fonction de la taille des accumulations de bois (Harmon et al., 1986) et de l'âge de la forêt riveraine (Evans et al., 1993). De plus, la présence d'une encoche d'érosion dépend de la position de l'embâcle dans le cours d'eau. Ces processus s'observent essentiellement dans des secteurs où la berge est fragilisée et lorsque les écoulements se concentrent dans un chenal limité.

Ces processus peuvent avoir des conséquences graves, en menaçant par exemple des infrastructures dans des zones urbanisées. Le cas le plus habituel est celui des voies de communication parfois mises à mal lors des crues.

Pourtant, ils ne constituent pas seulement un risque. Ils font partie des processus naturels caractérisant le fonctionnemént écologique des cours d'eau. En agissant sur les transferts de sédiments, la succession seuils-mouilles, les dimensions et la stabilité du chenal (Gurnell et al., 1995), ils augmentent entre autre l'hétérogénéité morphologique des hydrosystèmes. Par exemple, dans les cours d'eau de piémont à méandres actifs, les débris ligneux contribuent à accélérer les recoupements par débordement (Keller et Swanson, 1979 ; Piégay, 1993) et à préserver ainsi un plus grand nombre d'annexes fluviales. Sur d'autres rivières, les embâcles permettront de conserver une plus grande variabilité de la largeur du chenal (Sweeney, 1992; Nakamura et Swanson, 1993). C'est ainsi qu'en Angleterre, certains auteurs font état de programme de conservations patrimoniales et écologiques («Sites of Special Scientific Interest») de tronçons caractérisés par une érosion latérale active (Hooke, 1992 ; Newson et Sear, 1992 ; Gilvear, 1993).

Par ailleurs, les embâcles ne sont pas systématiquement facteurs d'érosion, ils peuvent au contraire participer à la stabilisation du chenal ou des berges. Sur des grands cours d'eau, les débris ligneux grossiers peuvent, par exemple, protéger certaines berges en favorisant la dissipation de l'énergie. Ainsi, Hickin (1984) a montré que les accumulations de bois parfois très importantes sur la berge de concavité de la rivière Squamish (rivière à méandres du Canada) ralentissaient considérablement la vitesse de recoupement. La technique du peigne décrite par Lachat (1994), où l'on entasse branches, troncs et arbres parallèlement au sens du courant pour protéger les berges sapées latéralement est un exemple appliqué des propriétés stabilisatrices d'un embâcle. Sur des petits cours d'eau de montagne, le bois mort participe à l'équilibre morphologique de la rivière en limitant le transfert des sédiments et en réduisant l'énergie hydraulique (Marston, 1982). C'est ainsi qu'à la suite de l'enlèvement d'embâcle sur ce type de cours d'eau, certains auteurs ont observé une déstabilisation du lit suivi de changements morphologiques importants (Heede, 1981 ; Bilby, 1984 ; Shields et Smith, 1992 ; Smith et al., 1993).

\section{II —'EMBÂCLE : PLUSIEURS FONC- TIONS ÉCOLOGIQUES}

\subsection{Des habitats pour la faune aquatique}

\section{- Diversification physique du milieu}

Les modifications hydrauliques liées à la présence d'embâcles, caractérisées par des variations locales des vitesses, du sens d'écoulement, des hauteurs d'eau et de la composition granulométrique, créent une grande variabilité physique du milieu. On obtient ainsi une mosaïque de combinaisons différentes de ces trois variables physiques (vitesse, hauteur, granulométrie) au niveau de ces structures (fig. 3). L'habitat des organismes aquatiques, dont la sélection dépend pour beaucoup de ces trois variables, se trouve alors largement enrichi, chaque espèce et stade pouvant rencontrer les conditions favorables à leur survie et leur développement.

A une échelle plus globale, ces structures participent à la diversification des formes fluviales. Dans des petits cours d'eau de montagne, les troncs tombés, en créant des escaliers où se succèdent mouilles et seuils, augmentent la diversité dẹs habitats (Bisson et al., 1987; Sedell et al., 1987). Sur des cours d'eau à fond sableux, seules les grosses structures ligneuses sont susceptibles de générer ce type de succession de faciès.

Dans des cours d'eau plus larges, ces accumulations ligneuses peuvent être à l'origine de mésoformes telles que des îles ou des bras secondaires qui vont créer des condi-

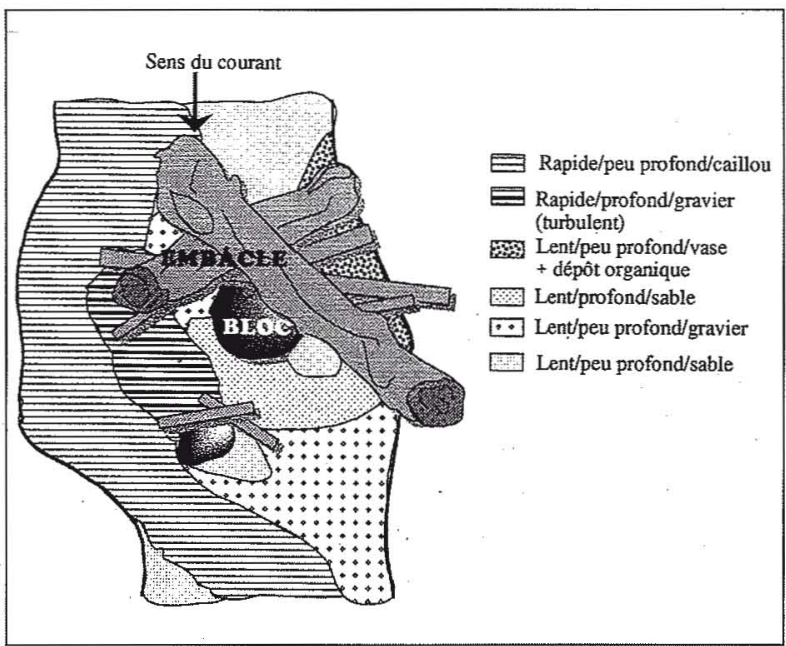

3. Les structures ligneuses, une mosaïque d'habitats. 
tions d'habitat diversifiées nécessaires au développement de la faune aquatique et terrestre.

- Utilisation de cet habitat par la faune

L'habitat conditionne la nature, la présence et la densité des organismes aquatiques présents dans un milieu (invertébrés, poissons). Autrefois perçus comme un obstacle à la migration des poissons ou comme une cause de la réduction de l'oxygène dans l'eau suite à un envasement, les embâcles sont aujourd'hui reconnus comme augmentant la qualité de l'habitat en complexifiant et diversifiant le milieu. Or plus les habitats sont hétérogènes et complexes plus la richesse spécifique des communautés vivantes augmente.

Ainsi, les débris ligneux grossiers et les microenvironnements qui leur sont associés constituent un habitat pour beaucoup d'animaux (Harmon et al., 1986; Gregory et Davis, 1992). Par exemple, certaines larves de Coléoptères (famille des Dryopidae) habitent les bois pourris en creusant des galeries tout en se nourrissant des débris organiques qu'elles libèrent; d'autres se fixent simplement sur ces structures (famille des Psychomyiidae).

Pour les poissons, les embâcles jouent le rôle d'abri, élé ment indispensable lors des périodes quotidiennes de repos. En ralentissant les vitesses d'écoulement, cet amalgame complexe formé par l'enchevêtrement des bois forme des caches: elles sont utilisées d'une part comme abris hydrauliques, où les poissons se maintiennent facilement sans dépenses d'énergie excessives, et d'autre part comme protection contre les prédateurs terrestres et aquatiques. Ainsi, en procurant des barrières visuelles qui limitent la compétition, ces structures peuvent accueillir un grand nombre d'espèces différentes (Angermeier et Karr, 1984).

Parallèlement, à l'échelle des faciès, les mouilles profondes associées à ces embâcles, qui disposent de couvert, jouent la même fonction de cache que les structures ligneuses proprement dites. Elles fournissent un site de repos pour la plupart des espèces en toutes saisons, surtout en période hivernale et de migration. En période d'étiage, sur les petits cours d'eau, ces mouilles contiennent des quantités d'eau importantes et durant les étés chauds conservent une eau plus frâche qui sert de refuge thermique aux poissons, particulièrement aux Salmonidés sensibles aux fortes températures (Bisson et al., 1987).

De même, afin de survivre à des conditions critiques d'écoulement et de températures lors de crues et d'hivers rudes, certains poissons gagnent des affluents du cours principal ou les annexes fluviales comme les bras secondaires (Tschaplinsky et Hartmann, 1983). Caractérisés par une plus grande diversité d'habitat liée à la présence plus importante d'embâcles, ces milieux jouent le rôle de refuge. Les zones humides annexes, où la production est importante en raison de l'absence de courant, servent également de zone de reproduction et de nurserie pour un grand nombre de poissons de ces zones aval (Roux et Copp, 1993).

\subsection{Une ressource trophique}

Les embâcles, en formant des obstacles, servent de pièges et de structures de rétention pour les particules détritiques dérivantes telles que les feuilles, les tiges ou d'autres troncs. Cet effet filtre sera d'autant plus important que le cours d'eau est de faible largeur. Sur des bassins versants boisés dans les montagnes du New Hampshire aux Etats-Unis, Bilby et Likens (1980) estiment que les débris ligneux piègent $75 \%$ de la matière organique dans des cour's d'eau de premier ordre, et respectivement $58 \%$ et $20 \%$ dans des cours d'eau de second et de troisième ordre.

Les débris ligneux sont largement colonisés par les invertébrés benthiques qui rencontrent dans ces amas organiques des conditions favorables pour se nourrir ou pondre. Cette communauté très riche sert de base alimentaire aux poissons qui trouveront dans ces sites des milieux propices à la croissance et au développement des jeunes stades. Par exemple, sur la Satilla, cours d'eau à fond sableux de plaine côtière du Sud-Est de l'Etat de Géorgie aux Etats-Unis, Benke et al. (1985) montrent que les bois morts sont potentiellement plus riches que les sables ou les vases tant en qualité (diversité des espèces) qu'en quantité (biomasse). Ils représentent seulement $4 \%$ de la surface totale habitable, mais abritent $60 \%$ de la biomasse d'invertébrés.

Par conséquent, les modifications des structures de rétention pourront induire des perturbations dans la dynamique des éléments nutritifs qui agiront à long terme sur les stocks piscicoles. En Alaska, Elliott (1986) constate immédiatement après l'extraction des embâcles du lit mineur une diminution de 60 à $90 \%$ du nombre total d'invertébrés et d'invertébrés dérivants. Les poissons sont alors soumis à une diète, qui peut provoquer dans certains cas l'affaiblissement et la mort d’individus très sensibles.

\section{III 國 QUELLE GESTION PRÉCONISER?}

\subsection{Intégrer le bois mort dans une démarche de réha- bilitation}

Dans les pays anglo-saxons, les gestionnaires reconnaissent aujourd'hui l'importance environnementale des débris ligneux grossiers dans les cours d'eau et souhaitent les promouvoir dans la réhabilitation des habitats piscicoles dégradés.

Aux Etats-Unis, des débris ligneux grossiers ont été volontairement préservés (Lisle, 1981) et parfois réintroduits (fig. 4) dans les rivières en raison de leur potentialité piscicole (Robison et Beschta, 1990) Newbury et Gaboury, 1993). Des troncs sont ainsi apportés d'autres secteurs. Ces opérations sont néanmoins con̂teuses et endommagent souvent les berges et le lit. Aussi, les ingénieurs prennent de

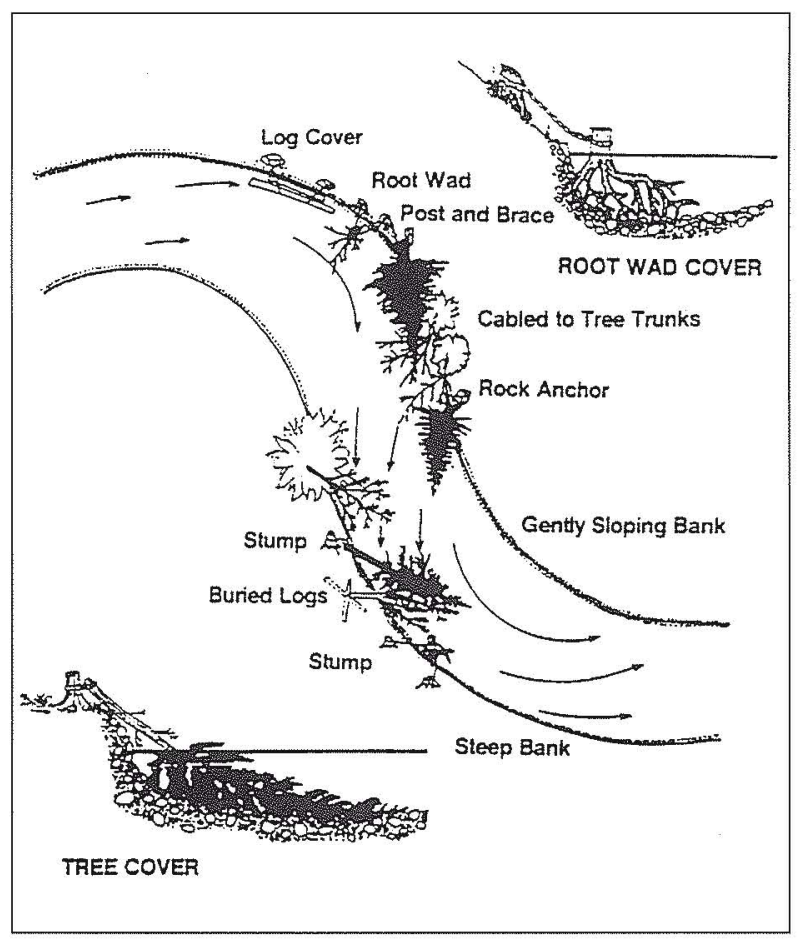

4. Réintroduire des débris ligneux grossiers afin d'augmenter l'habitat des salmonidés (d'après un « Stream Enhancement Guide » américain, Anonymes, 1980). 
plus en plus part à ces recherches afin de proposer des modèles destinés à évaluer les volumes disponibles in situ au cours du temps (Van Sickle et Gregory, 1990; Robison et Beschta, 1990), prévoir les effets hydrauliques de l'enlèvement, le déplacement, voire la réintroduction des débris dans les rivières (Gippel et al., 1994). La nécessité de définir des procédures objectives de gestion du bois dans les cours d'eau américains s'affirme donc aujourd'hui (Gippel et al., 1994).

En France, ce type de démarche n'est encore que balbutiante, l'Etat préconisant toujours l'entretien traditionnel et efficace de la végétation riveraine et des embâcles de bois afin de maintenir la capacité d'écoulement du lit. Néanmoins, certains éléments sont encourageants et confirment que cette politique devrait s'assouplir et surtout mieux s'adapter aux milieux d'intervention. Dans une plaquette intitulée "La végétation aux abords des rivières: source de vie et d'équilibre » (Maridet et Collin-Huet, 1995), le Ministère de l'Environnement met bien en évidence l'intérêt écologique des embâcles de bois. L'Article L 200-1 de la loi Barnier du 2 février 95 précise en outre que «... la diversité et les équilibres biologiques \{générées par les débris ligneux par exemple $\}. .$. font partie du patrimoine de la nation ». Enfin, la volonté de restaurer les milieux alluviaux particulièrement dégradés et de préserver le fonctionnement de milieux remarquables est souligné dans la plupart des grands bassins fluviaux (grandes orientations des SDAGE présentées en 1994). Or, une telle démarche nécessite de prendre en compte la dynamique du bois mort puisque les cours d'eau des plaines alluviales de l'Europe tempérée disposent naturellement d'un corridor boisé à l'origine de flux organiques grossiers.

\subsection{La notion de non-entretien}

Modifiant l'équilibre morphologique de la rivière, perturbant le fonctionnement écologique, l'enlèvement des embâcles n'est pas une action anodine. De même, comme nous l'avons vu précédemment, le nettoyage de la rivière n'est pas toujours la solution la mieux adaptée lorsqu'il s'agit 'de gérer l'inondation des secteurs aval. La logique économique est également discutable. En effet, les formations végétales riveraines des cours d'eau d'Europe sont dominées par l'arbre (Petts, 1989 ; Wenger et al., 1990) et alimentent donc en permanence le cours d'eau en débris ligneux. Par conséquent, une politique d'entretien devra être continue dans le temps. Si l'on retient un coût d'entretien en zone rurale de l'ordre de 5000 à 15000 francs $/ \mathrm{km}$ (Ministère de l'Environnement et Agences de l'Eau, 1985), une telle politique ne peut pas être menée à l'échelle du territoire national. Un coût moyen d'entretien de 10000 francs $/ \mathrm{km}$ sur la moitié seulement des rivières du bassin Rhône-Méditerranée-Corse (23,6\% de la superficie nationale), nécessite par exemple une enveloppe financière, reconduite tous les 10 ans environ de 750 millions de francs $(75000 \mathrm{~km}$ de cours d'eau à entretenir), soit 75 millions par an ce qui dépasse largement la somme de 5,5 millions de francs sur 10 ans proposée par le gouvernement le 24 janvier 1994 pour mener à bien cette tâche sur l'ensemble du territoire français. Ceci représente en outre 1,25 fois le budget 1994 de l'Agence de l'Eau RMC en matière de restauration de rivière alors que celle-ci subventionne actuellement l'entretien à hauteur de 5 millions de francs par an (soit 0,08\% de son budget; Piégay, 1995).

Assouplir la logique d'entretien lorsque celle-ci est envisagée, et réfléchir à son bien-fondé en tenant compte des spécificités du cours d'eau sur lequel une intervention est souhaitée apparaît nécessaire. Dans cette perspective, le non entretien peut être préconisé sans pour autant signifier que le gestionnaire se désintéressera de la rivière. Le non-entretien contrôlé peut être une réelle stratégie de gestion à la fois économique, écologique, patrimoniale et hydraulique.
D'un point de vue opérationnel, il est possible de considérer que l'enlèvement des embâcles est :

- nécessaire lorsque le risque d'inondation des terrains riverains est humainement et économiquement élevé, que la destruction d'ouvrages est reconnue ou lorsque la rivière représente un potentiel touristique et paysager,

- inutile, voire non recommandé, lorsque l'objectif recherché est un ralentissement dynamique des écoulements ou une optimisation écologique. Une réhabilitation piscicole peut ainsi préconiser le maintien d'embâcles de bois. Des opérations ont déjà été décrites aux Etats-Unis, Lisle (1981) à Jacoby Creek montre qu'un embâcle mobile et situé au centre du chenal, formé d'une souche et de débris associés, n'a pas été détruit lors de l'entretien de la rivière mais simplement déplacé et stabilisé en bord de berge afin de jouer un rôle écologique optimal,

- partiel et réfléchi quand les gestionnaires de la rivière recherchent un équilibre entre les différents usages et la préservation du milieu.

\subsection{Gestion des embâcles et logique d'entretien de la végétation riveraine}

On ne peut pas dissocier la gestion des embâcles de celle de la végétation riveraine. Le jardinage systématique de la ripisylve tel qu'il est aujourd'hui encore proposé est remis en question au profit d'une gestion plus souple (Piégay et al., 1994). Comme la gestion des embâcles, l'entretien de la végétation doit être sectorisé, spatialisé en fonction du degré d'emprise humaine. La démarche devra ainsi reposer sur un plan territorialisé et pluri-annuel d'entretien (fig. 5). Les opérations seront adaptées à des objectifs clairement définis, ceux-ci devant être logiques avec la vocation potentielle du milieu. Elles devront être modulables tant au niveau de l'espace concerné (longitudinalement et latéralement) que de l'intensité et de la fréquence de l'intervention. Le niveau d'entretien dépendra de la vulnérabilité des terrains riverains à l'inondation, de leur utilisation par les populations riveraines comme espace de loisirs, de la qualité écologique de la ripisylve (largeur du corridor, diversité des espèces et des unités végétales), du type de cours d'eau (taille, alluvial ou

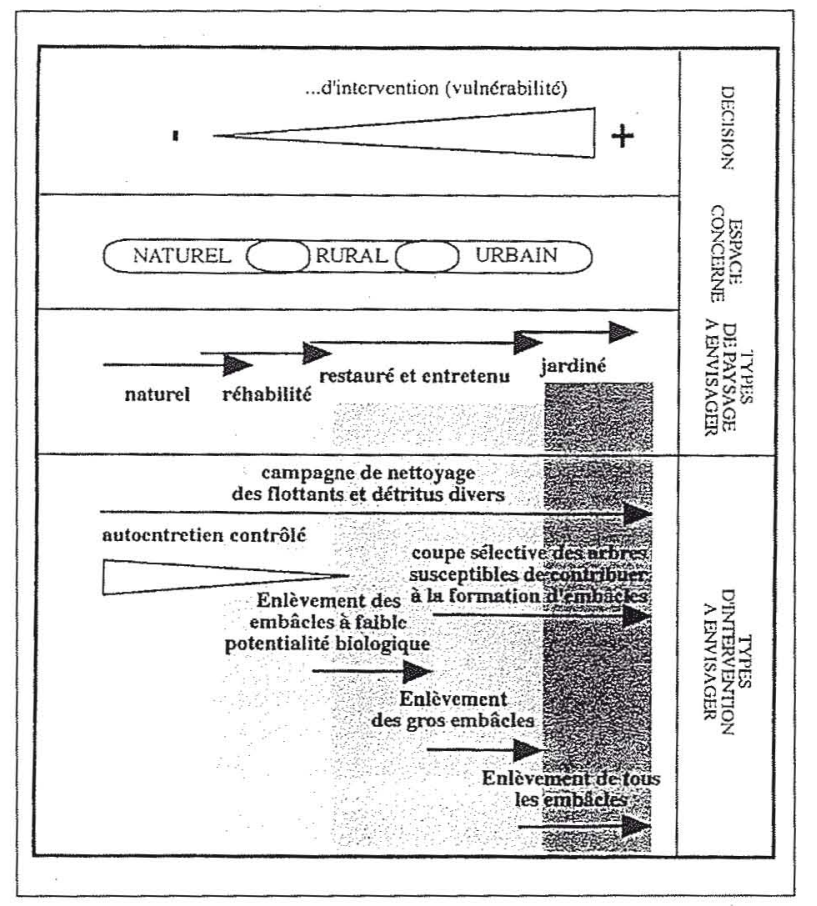

5. Une logique territorialisée de gestion des embâcles. 
non, énergie hydraulique élevée ou non). Très schématiquement, l'entretien sera d'autant plus important que les marges seront domestiquées donc vulnérables à l’inondation. On peut ainsi préconiser un entretien des cours d'eau (embâcles et ripisylve) limité à l'amont d'ouvrages et dans les zones fortement anthropisées, et une politique de non-intervention ou de renaturation de la végétation sur d'autres secteurs afin de retrouver un fonctionnement dans lequel les interconnexions lit mineur / lit majeur boisé seraient optimales. Tout en favorisant la diversification des habitats, la présence de cette végétation peut servir de filtre et piège aux bois flottants qui pourraient être source d'embâcles indésirables à l'aval.

Cette démarche territorialisée, aujourd'hui soutenue par l'Agence de l'Eau RMC, est en cours d'application sur la rivière Argens (convention « de gestion et d'aménagement de l'Argens » signée par la région PACA, le département du Var et l'Agence de l'Eau, 1991). "Une non-intervention contrôlée \{est prônée\} dans les secteurs sans enjeu de protection des personnes et des biens et sans gêne pour les usages. L'objectif est de laisser évoluer le cours d'eau et ses zones humides annexes avec leur propre capacité de régénération naturelle » (Thévenot et Saint-Léger, sous presse). De même, lors d'une expertise conduite à l'automne 1993 sur le Haut Roubion, une stratégie de gestion spatiale avait été proposée, trois types de tronçons étant identifiés. Le premier, écologiquement riche et peu soumis à la pression d'aménagement, ne devait pas être entretenu. Le second était occupé par des ouvrages ou des aménagements à préserver. Un entretien sévère de la végétation était proposé sur une longueur de $200 \mathrm{~m}$ environ à l'amont de chaque ouvrage ce qui devait permettre d'augmenter localement la section d'écoulement tout en maintenant une végétation arborée qui sert de piège potentiel aux ligneux en transit avant qu'ils ne forment un embâcle au niveau de l'ouvrage lui-même. Sur le dernier type de tronçon, l'intervention était plus souple. Au fur et à mesure de l'éloignement des zones à risque, la largeur à entretenir devait progressivement être réduite, l'entretien se limitant finalement au seul enlèvement des principaux troncs bloquant l'écoulement.

Ce type de démarche devrait permettre de conserver des secteurs en renaturation, écologiquement complexes et diversifiés, de sélectionner les sites où l'intervention est prioritaire afin de gérer plus efficacement le risque d'inondation. Cette approche est économiquement et stratégiquement nécessaire lorsque l'on sait que la déprise rurale actuelle conduit à un abandon croissant des espaces agricoles riverains voués à terme à un reboisement. Cette réflexion en matière d'entretien de cours d'eau n'est pas nouvelle en Europe. Les danois ont évalué les conséquences écologiques de telles interventions dès le début des années 1980 et ont dès lors modifié leurs pratiques d'entretien (Iversen et al., 1993).

\section{CONCLUSION}

Par l'intérêt écologique des embâcles et face au faible risque qu'ils représentent (érosion, inondation), il convient de développer une réflexion sur de nouvelles bases prenant en compte «les équilibres naturels des écosystèmes» (cadre de la loi sur l'eau de 1992). En effet, comme nous l'avons vu précédemment, les embâcles de bois influencent largement le fonctionnement écologique des cours d'eau. En agissant sur la morphologie, ils engendrent une grande diversité physique dans le milieu (hydraulique, granulométrique) et créent des conditions d'habitat propice au développement de la faune aquatique. Ils constituent également un piège à matière organique et une source de nourriture pour certains organismes.
Par ailleurs, ils ne peuvent être assimilés systématiquement à un risque d'érosion ou d'inondation.

Ainsi, dans le cadre de nouvelles orientations de gestion de ces structures ligneuses, il parait important de distinguer clairement les embâcles pérennes, qui induisent une diversification des paramètres physiques des écoulements (hauteur d'eau, vitesse, etc...) et les embâcles accidentels qui ne se produisent qu'en cas de crue. Les premiers ont souvent une incidence hydraulique faible et sont le plus fréquemment situés dans des zones peu sensibles au risque. Ils peuvent jouer un rôle bénéfique lors des épisodes de crue en favorisant le piégeage dans ces zones des matériaux transportés par l'eau du fait des conditions hydrauliques modifiées qu'ils induisent. En revanche, les embâcles accidentels dans les zones vulnérables peuvent avoir des conséquences néfastes, notamment en matière de risque. Des mesures de prévention peuvent limiter ces risques en assurant un entretien raisonné de la végétation en amont immédiat de ces zones vulnérables et en gérant rationnellement l'occupation du sol des zones concernées.

Une telle démarche territorialisée permettra d'aboutir à « une gestion équilibrée de la ressource en eau », en assurant sa valorisation économique de manière «à satisfaire les différents usages » et à maintenir un bon «fonctionnement écologique des écosystèmes aquatiques » (loi sur l'eau de 1992).

\section{Références}

[1] ANgermeier P.L. \& Karr J.R. (1984). - Relationships between woody debris and fish habitat in a small warmwater stream. Transactions of the American Fisheries Society; 113 , 716-726.

[2] Benke A.C., Henry R.L., Gillespie D.M. \& Hunter R.J. (1985). - Importance of snag habitat for animal production in southeastern streams. Fisheries, 10(5), 8-13.

[3] Bilby R.E. (1984). - Removal of organic debris may affect stream channel stability. J. of Forestry, 82(10), 609-613.

[4] Bilby R.E \& Likens G.E. (1980). - Importance of organic debris dams in the structure and function of stream ecosystems. Ecology, 61, 1107-1113.

[5] Bisson P.A., Bilby R.E., Bryant M.D., Dolloff C.A., Grette G.B., House R.A., Murphy M.L., Koski K.V. \& SEDELL. J.R. (1987). - Large woody debris in forested streams in the Pacific Northwest: past, present and future. In : Streamside Management : Forestry and Fishery Interactions, Seattle, College of Forest Resources, University of Washington, 143-190.

[6] Bisson P.A., Quinn T.P., Reeves G.H. \& Gregory S.V. (1992). - Best management practices, cumulative effects, and long-term trends in fish abundance in pacific northwest river systems. In : Naiman R.J. (eds), Watershed management - Balancing sustainability and environmental change. Springer-Verlag, New York, 189-232.

[7] Church M. (1992). - Channel morphology and typology. In : Calow P. and Petts G.E. (eds), The rivers handbook. Blackell Scintific Publications, Oxford, 126-143.

[8] ELLiotT S.T. (1986). - Reduction of a Dolly varden population and macrobenthos after removal of logging debris. Transactions of the American Fisheries Society, 115, 392-400.

[9] Evans B.F., Townsend C.R. \& Crowl T.A. (1993). - Distribution and abundance of coarse woody debris in some southern New Zealand streams for contrasting forest catchments, New Zealand J. of Marine and Freshwater Research, 27, 227-239.

[10] Gilvear D.J. (1993). - River management and conservation issues on formerly braided river systems ; the case of the river Tay, Scotland. In : Best J.L. and Bristow C.S. (eds), Braided Rivers. Geological Society Special Publication $n^{\circ} 75,231-240$ 